\title{
Mitohormesis in muscle cells: a morphological, molecular, and proteomic approach
}

\author{
Elena Barbieri \\ Piero Sestili \\ Luciana Vallorani \\ Michele Guescini \\ Cinzia Calcabrini \\ Anna Maria Gioacchini \\ Giosuè Annibalini \\ Francesco Lucertini \\ Giovanni Piccoli \\ Vilberto Stocchi
}

Department of Biomolecular Sciences, Division of Exercise and Health Sciences, University Carlo Bo, Urbino, Italy

Corresponding author:

Elena Barbieri

Department of Biomolecular Sciences, Division of Exercise and Health Sciences

University Carlo Bo

Via I Maggetti, 26

61029 Urbino, Italy

E-mail: elena.barbieri@uniurb.it

\section{Summary}

Low-level oxidative stress induces an adaptive response commonly defined as hormesis; this type of stress is often related to reactive oxygen species (ROS) originating from the mitochondrial respiratory chain (mitochondrial hormesis or mitohormesis). The accumulation of transient low doses of ROS either through chronic physical activity or caloric restriction influences signaling from the mitochondrial compartment to the cell, reduces glucose metabolism, induces mitochondrial metabolism, increases stress resistance and ultimately, increases lifespan. Mitochondrial formation of presumably harmful levels (chronic and/or excessive) of ROS within skeletal muscle has been observed in insulin resistance of obese subjects, type 2 diabetes mellitus, as well as in impaired muscle function associated with normal aging. Advances in mitochondrial bioimaging combined with mitochondrial biochemistry and proteome research have broadened our knowledge of specific cellular signaling and other related functions of the mitochondrial behavior. In this review, we describe mitochondrial remodeling in response to different degrees of oxidative insults in- duced in vitro in myocytes and in vivo in skeletal muscle, focusing on the potential application of a combined morphological and biochemical approach. The use of such technologies could yield benefits for our overall understanding of physiology for biotechnological research related to drug design, physical activity prescription and significant lifestyle changes.

KEY WORDS: mitochondria, skeletal muscle, ROS, hormesis.

\section{Introduction}

Mitochondria are dynamically involved in several muscle cellular activities including signaling, proliferation, differentiation, autophagy and death. Skeletal muscle cells, as well as the metabolically active neurons, accumulate the highest number of mitochondria. The dynamics, size, number and location of mitochondria in muscle cells vary significantly according to the cellular conditions (muscle fiber types, differentiation level, training, nutritional state) and energetic needs (contraction, steady-state, recovery). For instance, mitochondria accumulate predominantly at high energy demanding sites, close to calcium release units in striated muscle, providing a physical basis for localized Sarcoplasmic Reticulum - mitochondrial $\mathrm{Ca}^{2+}$ signaling and suggesting that mitochondria participate in $\mathrm{Ca}^{2+}$ cycling at the triad level ${ }^{1}$. Recently, the identity of mitochondrial $\mathrm{Ca}^{2+}$ transporters has been revealed, opening new perspectives for investigation and molecular intervention ${ }^{2}$. Given the role of mitochondria in a wide range of cellular processes, it is not surprising that mitochondrial damage has been implicated in the pathogenesis of several muscle as well as neurodegenerative or chronic diseases ${ }^{3,4}$. Mitochondria are generally considered as the main source of reactive oxygen species (ROS) in skeletal muscle cells. It is known that $2-5 \%$ of the total oxygen consumed by mitochondria may undergo one electron reduction with the generation of superoxide. Complexes I and III of the electron transport chain seem to be the main sites of mitochondrial superoxide generation ${ }^{5,6}$. Several physiological and pathological conditions may result in ROS production by mitochondria. For example, high intense contractile activity, disuse muscle atrophy or inflammation, increased mitochondrial ROS generation?. Although the excessive ROS production is associated with the aetiopathology of several human diseases, low levels of ROS are important mediators for a variety of cellu- 
lar processes, including cell adhesion, immune response, apoptosis, cell growth and differentiation. ROS also act as second messengers in intracellular signaling. In fact, it has become apparent that low levels of $\mathrm{H}_{2} \mathrm{O}_{2}$ may be required for sustaining cellular functioning and intracellular signaling. Transient increasing levels of ROS play a crucial role in modulating redox-sensitive gene expression, cell-signaling pathways and in preserving contractile function 8,9 However, chronic and excessive ROS production may lead to muscle cell damage and death. The prevalence of the role of ROS either in promoting benefits or establishing unfavorable conditions for the pathogenesis of a several skeletal muscle disorders or decline in muscle function, depends on the concurrence of intrinsic and extrinsic factors. These factors include the level and duration of ROS targeting muscle cells, the source, the site of ROS generation, the antioxidant status of target cells, the DNA repair capacity, the differentiation stage of muscle cells and the proliferative and myogenic capacity of satellite cells. Due to their hormetic nature, in muscle tissue mitochondrial ROS may trigger different signaling pathways leading to different and diverging responses, from adaptation to cell death. A significant number of studies describes the role of ROS in triggering many signaling pathways relevant to skeletal muscle cells' homeostasis and adaptation. Herein we will elucidate some of the signaling pathways triggered by ROS in mitochondrial muscle remodeling and their physio-pathological implications. Particular attention will be focused on advances in morphological and biomolecular approaches combined with mitochondrial proteome research in mitochondrial muscle remodeling in response to oxidative stress and the direct link between mitochondrial disruption and several biological modulations.

\section{Morphological methods}

\section{Bio-imaging}

Mitochondrial dynamics is a recent designation of mitochondrial behavior. Recent studies have led to renewed interest in determining the genes, proteins, and mechanisms that regulate mitochondrial shape, size, number, and distribution. Studies on mitochondrial live remodeling have mostly been performed using fluorescence microscopy ${ }^{10}$. This technique focuses on the development of fluorescent microscopy and specific fluorescent probes to stain mitochondria or specific mitochondrial molecules. Recent methodological approaches in fluorescent imaging technologies have improved our capacity to study both the dynamics and morphology of mitochondria by following specific metabolites and ions within mitochondrial sub-compartments, such as the membranes and matrix. A high number of fluorescent probes and dyes are increasingly used to quanti-qualitatively evaluate overall muscle mitochondrial number, membrane potential, oxida- tive stress, apoptosis, and $\mathrm{Ca}^{2+}$ concentrations ${ }^{10}$. Most of the mitochondrial dyes are taken up by the mitochondrial membrane potential. Such fluorescent dye markers may stain the structure of whole organelles, as well as mitochondrial parameters, such as redox potential, $\mathrm{Ca}^{2+}$ and $\mathrm{Cl}^{-}$levels, and $\mathrm{H}^{+}$relevant to the organelle functionality ${ }^{10}$. The dye fluorescence variations may depend on the surrounding environment and can also be used to measure the mitochondrial membrane potential, mass/volume, or oxidative state conditions in living cells.

\section{Oxidative level inside mitochondria}

ROS are very reactive molecules and extremely unstable, thus it is impossible to image them directly. The ROS detection levels have mainly relied on detecting their end products by chemiluminescence or by fluorescence. These products are usually formed when specific compounds react with ROS. One of the most frequently used techniques for detecting intracellular ROS, in particular hydrogen peroxide, depends on oxidation of the non-fluorescent substrate, such as 2,7-dichlorodihydrofluorescein a green fluorescent dye ${ }^{11,12}$. Due to the existence of several substances that interfere with the formation of 2,7-dichlorofluorescein (DCF), this probe, when used in cellular systems, is more useful as a marker of cellular oxidative stress ${ }^{11}$. Cell membranes are permeable to esterified forms of DCF. These products can easily enter cells and through deacetylation by esterases, they remain intra-cellularly trapped. The oxidation rate may be detected by a fluorimeter, fluorescence microscopy or by flow cytometry. Dihydroethidium (DHE) freely permeates cell membranes; thus, it is used extensively to monitor superoxide production. It had long been assumed that DHE, after reaction with superoxide anions, forms 2-hydroxyethidium a DNA intercalates which shows a red fluorescent product ${ }^{12}$. DHE is possibly the most specific, it reveals essential superoxide radicals, is preserved well by cells, and may also stand mild fixation ${ }^{12}$. The fluorophore10N-nonyl acridine orange (NAO) tightly binds to the mitochondrial cardiolipin in mitochondrial membranes in eukaryotes and bacteria. Cardiolipin content may decrease in oxidative stress conditions. The cardiolipin-sensitive probe NAO may be used to monitor oxidative changes in mitochondrial lipids and indirectly indicates the mitochondrial peroxidation level ${ }^{13}$.

\section{Mitochondrial ultrastructure}

Electron microscopy has enormously improved its application during the last few decades and it has become a powerful tool for studying mitochondria ultrastructure and function. Various modifications may be taken into consideration for sample preparation in fixation, dehydration, sectioning, inclusions and staining of the section to maintain the original morphology. In our investigations undifferentiated C2C12 
myoblast and differentiated myotube monolayers were washed and fixed with $2.5 \%$ glutaraldehyde in $0.1 \mathrm{M}$ phosphate buffer for $15 \mathrm{~min}$ to maintain their morphological structure. They were then gently scraped and centrifuged at $1200 \mathrm{rpm}$. Cell pellets were further fixed with glutaraldehyde for $1 \mathrm{~h}$. All specimens were $\mathrm{OsO}_{4}$ post-fixed, alcohol dehydrated, and embedded in araldite. Thin sections were stained with uranyl acetate and lead citrate. Mitochondrial density was calculated in 20 different areas of $10 \times 15 \mathrm{~cm}$ at 28000 magnification. Mitochondrial sizes were evaluated at 28000 magnification using the Philips CM10 microscope and Megaview software system ${ }^{14}$. Data from our group ${ }^{15}$ indicate that a mildly toxic $\mathrm{H}_{2} \mathrm{O}_{2}$ treatment during the early stages of C2C12 myoblast differentiation results in GSH depletion and strongly impairs the differentiative outcome. Interestingly, $\mathrm{H}_{2} \mathrm{O}_{2}$-injured cells showed signs of extensive mitochondrial degeneration (swelling and disruption) and lower mitochondrial density, suggesting that these organelles are specifically targeted by - or particularly sensitive to - exogenous ROS. Loss of mitochondria is a clearly detrimental event in a process typically requiring active mitochondriogenesis such as muscle differentiation ${ }^{16}$. These effects are visible by the inserts in the TEM micro- graphs of $\mathrm{H}_{2} \mathrm{O}_{2}$-treated cells at both differentiation day (DifD) 3 and 5 of Figure 1.

\section{Mitochondrial subcellular localization}

In striated muscle, the subcellular localization of all intracellular mitochondria largely depends on myofiber organization and plasma membrane invaginations of the $\mathrm{T}$ tubules. Interactions of mitochondria with the sarco-endoplasmic reticulum (SR/ER) have been receiving a great deal of attention because of emerging evidence on the role this site has in cell signaling and mitochondrial dynamics.

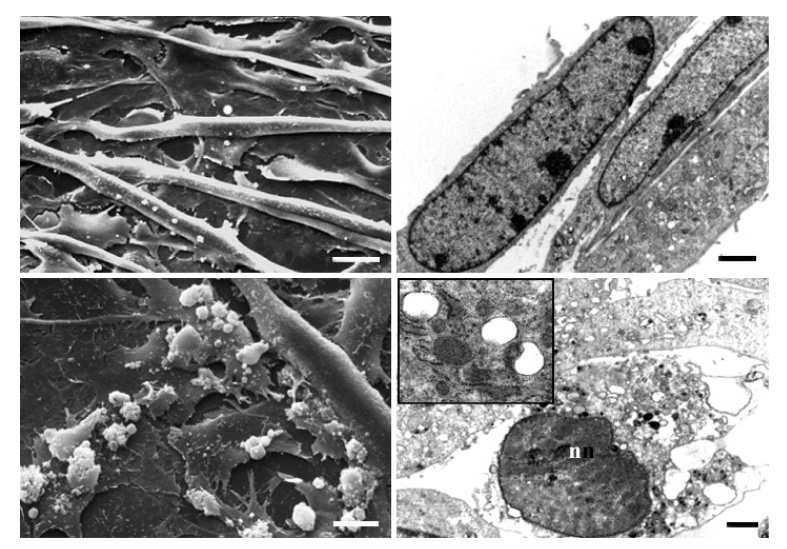

Mitochondria respond to different stimuli through remodeling, thus they pass from a dynamic behavior to a stabilized condition. In fact, they structurally localize in precise subcellular positions probably anchored with the face of the junctional SR opposite to that of the type 1 ryanodine receptor (RyR1) feet. This close association and tight coupling between the outer mitochondrial membrane (OMM) and the calcium releasing unit (CRU) in muscle is supposed to respond in the local domain of calcium signaling and metabolic coupling during normal muscle activity. The contractile activity of muscle has to be matched by mitochondrial ATP generation that is completed, at least in part, by the propagation of $\mathrm{Ca}^{2+}$ signals from SR to mitochondria. Muscle has a highly geometrical structure, providing only limited opportunity for mitochondrial $d y$ namics and inter-organellar interactions. Mitochondria are closely tethered to the SR terminal cisternae ${ }^{17}$, the RyRs are localized at some distance from mitochondria (about 100nm) as these $\mathrm{Ca}^{2+}$ release channels are located on the SR surface facing the T tubules ${ }^{18}$. Mitochondrial $\mathrm{Ca}^{2+}$ uptake in muscle depends on the diffusion of $\mathrm{Ca}^{2+}$ released from the $\mathrm{SR}$ to the mitochondrial surface ${ }^{18}$.

Triad-targeted mitochondria in adult skeletal muscle are remarkably stable and immotile. Because CRUmitochondrion $\mathrm{Ca}^{2+}$ cross-talk in skeletal muscle operates via local signaling microdomains, this "privileged" signaling could best be ensured by restricting mitochondria to a location immediately adjacent to sites of $\mathrm{Ca}^{2+}$ release by a strong structural anchoring mechanism (Fig. 2). Mitofusin 2 is probably the main player in the formation of such ER/mitochondrial close appositions. Mitofusin 2 is localized both in the outer mitochondrial membrane and on the ER surface ${ }^{19}$.

Of particular interest is the recent discovery of SR/ER-mitochondria tethers that are formed by multiple proteins, and local $\mathrm{Ca}^{2+}$ transfer between SR/ER and mitochondria. Given that the molecular identities of mitochondrial $\mathrm{Ca}^{2+}$ uniporter (MCU) has been un-

Figure 1. Differentiating C2C12 at SEM and TEM: the effect of oxidative challenge. Micrographs were taken at DifD 3 and 5. Initial thin myotubes appeared in control cells at DifD3, while an increased number of thicker and longer myotubes were present at DifD5. $\mathrm{H}_{2} \mathrm{O}_{2}$ induced necrosis at DifD3 and DifD5 (n). The inserts in the TEM micrographs of $\mathrm{H}_{2} \mathrm{O}_{2}$-treated cells at DifD3 and 5 highlight mitochondrial swelling and disruption caused by oxidative stress. Bars: SEM $10 \mathrm{Im}$; TEM $1 \mathrm{Im}$. (Adapted from Sestiliet al. ${ }^{15}$, with Authors permission). 

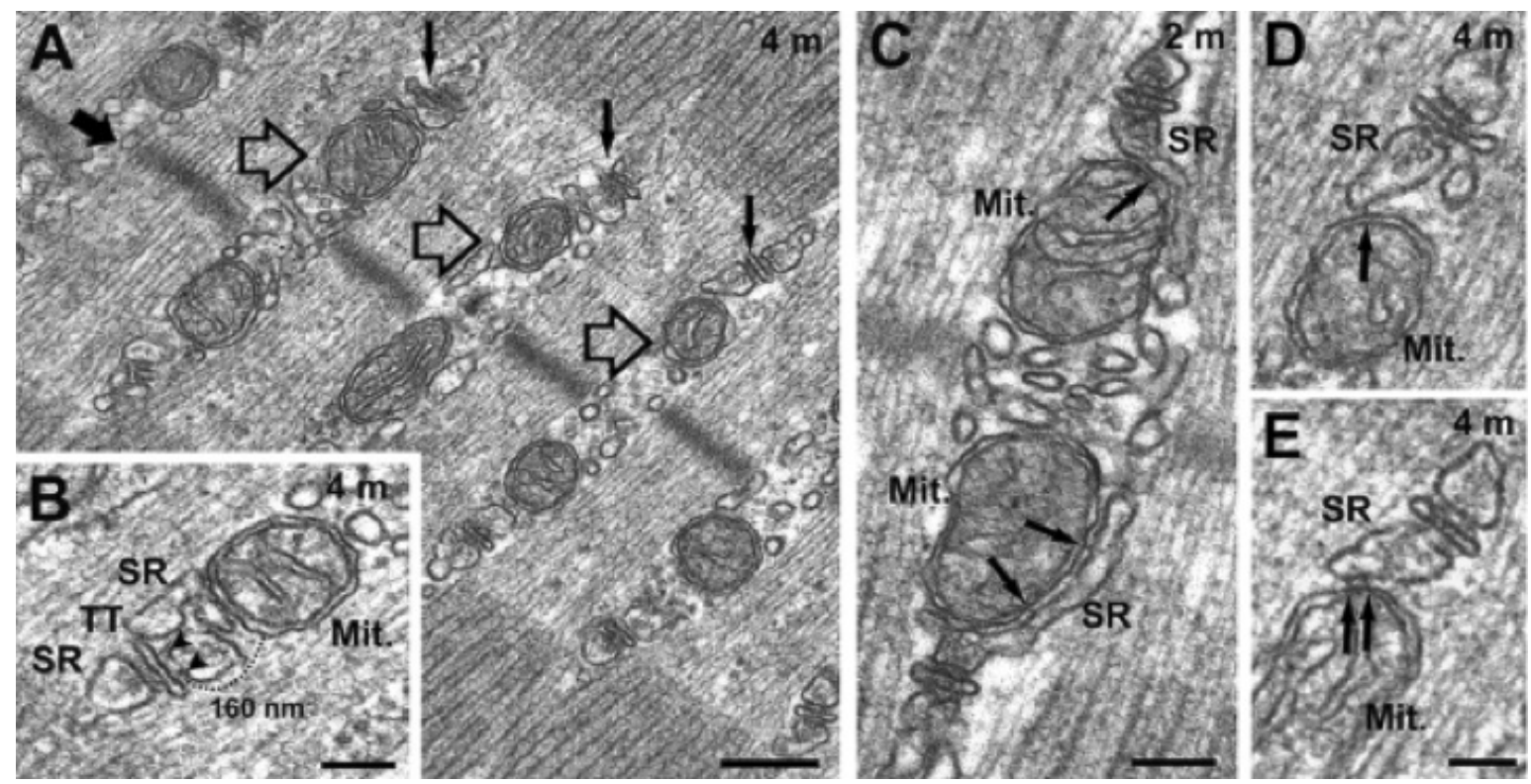

Figure 2. Mitochondria and CRUs are close to each other and connected by small electron dense strands, or tethers. (A) In adult muscle fibers, triads (small black arrows) are located in proximity to the sarcomere A-I band junction. Mitochondria (open arrows) are adjacent to the triad, on the side opposing the Z-line (large black arrow). (B) Mitochondria associated with the SR terminal cisternae are on the side opposite to that of the $\mathrm{Ca}^{2+}$ release channels, or RyRs (arrowheads). The dashed line shows the shortest distance between release sites (RyR-feet) and the outer mitochondrial membrane. (C-E) Under higher magnification, small electron-dense strands (black arrows) appear to tether, or bridge, individual mitochondria (Mit) to the triad. This structural linkage occurs specifically between the mitochondrial outer membrane and the SR terminal cisterna, generally on the side opposite to that facing the T-tubule ( $D$ and $E$ ), but also between mitochondria and the lateral sacks and tubules of the SR that surround the mitochondrion (C). Bars, (A) $0.25 \mathrm{~m}$; (B and C) $0.1 \mathrm{~m}$; (D and E) $0.05 \mathrm{~m}^{(\mathrm{Copyright}}{ }^{\circ}$ 2009 by The American Society for Cell Biology with permission from Boncompagniet al. ${ }^{1}$ ).

raveled only very recently ${ }^{2}$, the information on the tissue expression of these molecules is still fragmentary and the identity of the specific complex of anchoring that stabilizes the Mitochondrial-Sarcoplasmic Reticulum is still unknown.

In skeletal muscle, mitochondria occur as two sub-cellular populations identified as subsarcolemmal (SS) and intermyofibrillar (IMF) mitochondria (Fig. 3). Biochemical and functional differences between IMF and SS fractions exist, and they probably represent mitochondria at different stages of maturation or biogenesis. Changes may not only be due to mitochondrial protein synthesis but also to nuclear-directed protein synthesis exclusive to each mitochondrial population.

It has been described how acute contractile activity exerts differential effects on protein turnover in IMF and SS mitochondria, and it appears that intra-mitochondrial protein synthesis does not limit the extent of chronic contractile activity-induced mitochondrial biogenesis ${ }^{20}$. These mitochondrial subpopulations respond differently to various physiological perturbations. For example, SS mitochondria appear to be more malleable in response to exercise and pharmacological interventions. It seems possible that SS mitochondria possess more sensitive transcriptional control of their mitochondrial genome, although this concept has yet to be investigated.

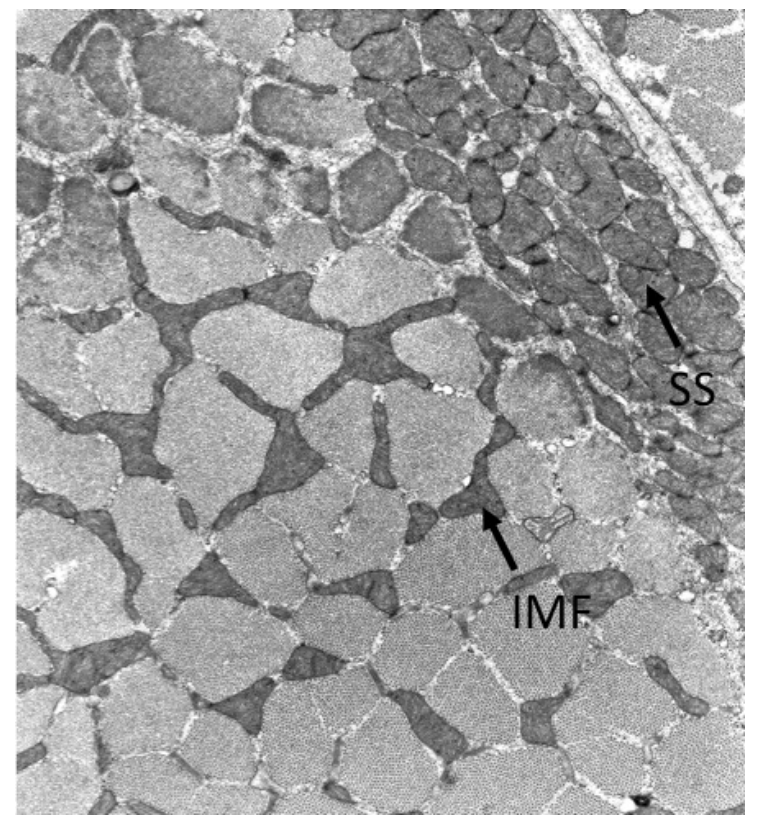

Figure 3. Electron micrographs illustrating the two mitochondrial populations in mice gastrocnemius muscle. Black arrows indicate mitochondria: subsarcolemmal (SS) and intermyofibrillar (IMF) mitochondria ( $\times 50,000$ magnification, Courtesy of Professor David Hood, University of York). 


\section{Molecular and biochemical methods}

\section{Mitochondrial life cycle signaling}

Most of the molecular studies on muscle mitochondria have relied on nuclear control of mitochondrial function. This approach has yielded important findings over the last few decades with the discovery of multiple transcription factors and cofactors governing mitochondrial dynamics, biogenesis and crosstalk with the nucleus. Furthermore, mitochondria are integral to other components of stress response or quality control including ROS signaling, unfolded protein response and mitochondrial autophagy. The main targets of mitochondrial signaling controlling the biogenesis, dynamicity and mitophagy of these organelles in muscle are summarized in Figure 4.
The mitochondrial life cycle starts with biogenesis, then mitochondria, through repeated cycles of fission and fusion, organize themselves into two distinct possible conditions: discrete units and networks. Finally, mitochondria life cycle ends with degradation of impaired or surplus organelles by mitophagy (turnover), which involves different biological mechanisms such as mitoptosis, apoptosis and/or autophagy, depending on the physio-pathological conditions ${ }^{21,22}$. In particular, the mitochondrial biogenesis signaling activated by peroxisome proliferator-activated receptor gamma coactivator alpha 1 (PGC-1) members family involves the transcription factors that regulate the expression of nuclear genes such as nuclear respiratory factor (NRF)-1/2 and estrogen-related receptor- $\alpha$ (ERR- $\alpha$ ). These three latter genes control the expression of nuclear genes encoding mitochondrial proteins and in-

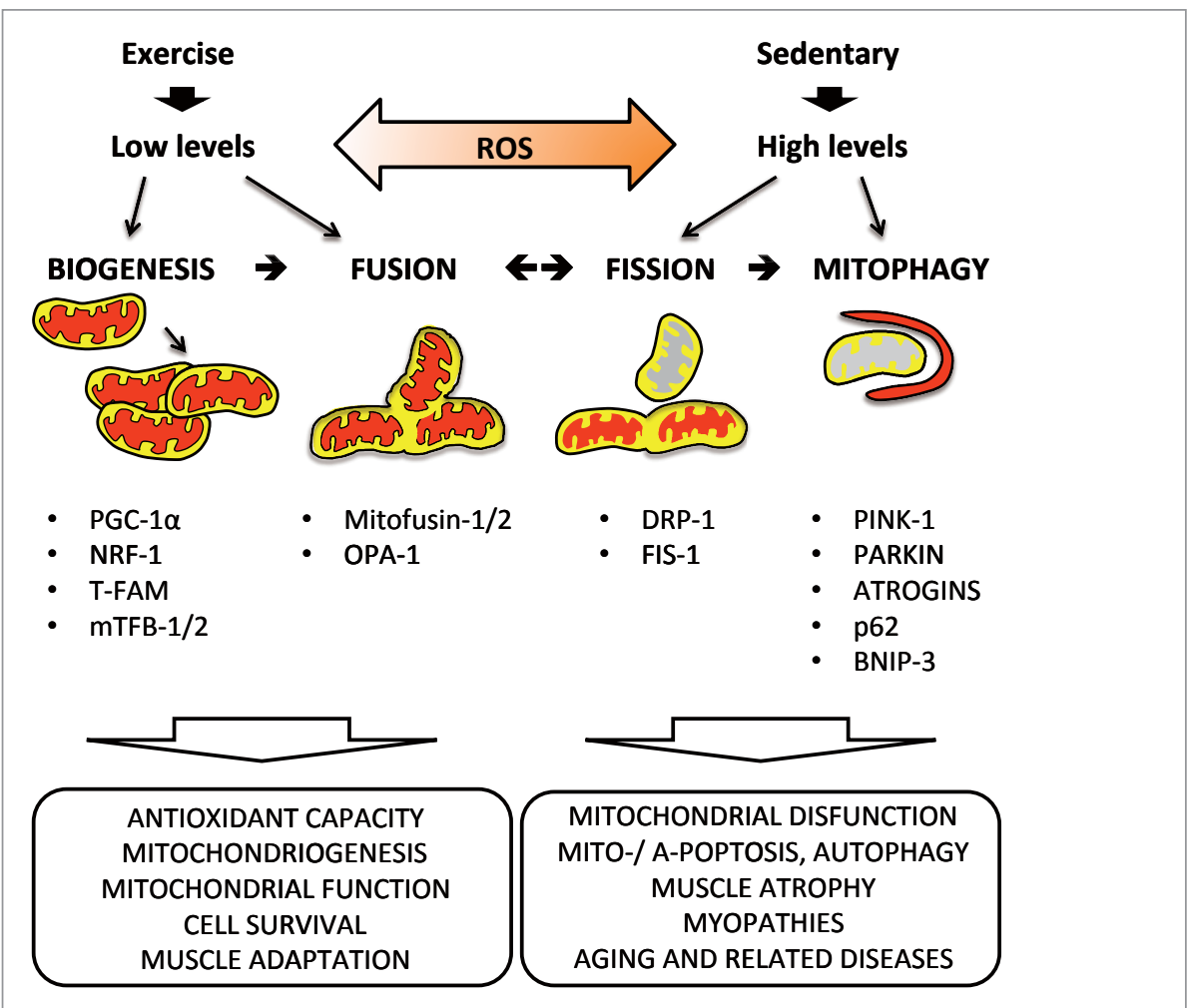

Figure 4. Mitohormesis and principal signaling pathways activated in skeletal muscle for mitochondrial biogenesis, remodeling, mitophagy and metabolic activation. Low levels of ROS activate mitochondrial biogenesis and mitochondrial remodeling through the action of the key regulators PGC-1a, NRF-1, T-FAM, mTFB-1/2, Mitofusin-1/2 and OPA-1. As well as, antioxidant enzymes that function as back-regulators of intracellular ROS levels. These molecules activate cellular mechanisms for muscle adaptation promoting oxidative metabolism and endogen antioxidants which may prevent oxidative damage and potential chronic diseases. Conversely, high levels of ROS determine mitochondrial damage that leads to fission (DRP-1 and FIS-1) and subsequent mitophagy (PINK-1, PARKIN and BNIP-3), mitoptosis or even cell death, when removal of impaired mitochondria together with antioxidant mechanisms are not sufficient to counteract increasing ROS exposure myopathies and aging related diseases develop.

It is challenging to clearly define the ROS dose required to promote the differing physio-pathological responses, the limits between the double face of ROS actions is still not clear.

BNIP-3, Bcl-2/adenovirus E1B 19-kDa interacting protein 3; DRP-1, dynamin-related protein 1; FIS-1, mitochondrial fission protein 1 ; m-TFB, mitochondrial transcription factor B; NRF, nuclear respiratory factor; OPA-1, optic atrophy 1; p62, polyubiquitin binding protein; PGC-1 $\alpha$, peroxisome proliferator-activated receptor gamma coactivator 1 alpha; PINK-1, PTEN-induced putative kinase 1; ROS, reactive oxygen species; T-FAM, mitochondrial transcription factor A. 
duce expression of mitochondrial transcription factor $A$ (T-FAM) and B 1/2 (mTFB), which regulates mtDNA replication and transcription, thus activating the coordinated expression of mitochondrial proteins ${ }^{14}$.

Mitochondrial fusion and division are modulated by GTP-hydrolyzing proteins (GTPases) belonging to the dynamin superfamily GTPases: mitofusins 1/2 control outer mitochondrial membrane fusion, optic atrophy 1 (OPA-1) mediates inner mitochondrial membrane fusion and dynamin-related protein 1 (DRP-1) regulate the division of outer and inner mitochondrial membranes ${ }^{23}$.

Recent work provides evidence for specific targets that control the mitochondrial turnover in the course of autophagy-dependent degradation of damaged or depolarized mitochondria such as: PTEN-induced putative kinase 1 (PINK-1), a protein that stabilizes mitochondrial integrity and function, important for the maintenance of mitochondrial networks ${ }^{24}$; Parkin, which is selectively recruited from the cytoplasm to damaged mitochondria by PINK-1 and senses damaged mitochondria for compensatory clearance by autophagy, is ${ }^{25}$ also involved ${ }^{26}$. In addition, Bcl-2/adenovirus E1B 19-kDa interacting protein 3 (BNIP-3) is a proapoptotic member of the Bcl2 family that seems to participate directly in mitophagy inducing the mitochondrial translocation of DRP-1. Notably, DRP-1-mediated mitochondrial fission is correlated with increased autophagy ${ }^{27}$. Other target genes are involved in non selective autophagy, as Atrogins and the polyubiquitin binding protein $p 62^{22}$.

When compromised by various kinds of damage, solitary mitochondria undergo mitophagy, a self-regulated eating process that plays key roles in multiple muscle cell activities. Interplay between mitochondria and autophagy seems to be evolutionarily conserved ${ }^{28}$. Defects in autophagic degradation selective for mitochondria are associated with neuromuscular diseases, emphasizing their physiological relevance to cellular functions. In response to mitophagy mitochondria fragment. This morphological change is accomplished by molecular signals, resulting in the capture of the organelle by the autophagosomes. By contrast, during macroautophagy mitochondria fuse in order to avoid degradation and to sustain ATP production in times of limited nutrient availability 25 . The dynamin-related GTP ases OPA-1 of the inner mitochondrial membrane, and mitofusin 1 and 2 of the outer membrane, control mitochondrial fusion in mammalian muscle cells. Fission of mitochondria is controlled by DRP-1. Translocation of DRP-1 from the cytoplasm to mitochondria is a crucial step for fragmentation of the organelle and depends on the Ser 637 residue dephosphorylation by calcineurin. By contrast, Ser637 phosphorylation by PKA induces mitochondrial elongation. DRP-1 inside the mitochondria can be stabilized by SUMO ylation mediated by SUMO ligases, which explains how dynamic regulation of fission may adapt mitochondrial morphology to cellular demands ${ }^{25}$. A growing body of evidence suggests that autophagy of mitochondria is a selective and defense-oriented response against ROS, mitochondrial dysfunction, and the accumulation of somatic mutations of mtDNA associated with aging ${ }^{29}$; damaged mitochondria are removed by mitophagy by BNIP-3 and mitochondrial fission protein 1 (FIS-1), a pro-fission mitochondrial protein that induces mitochondrial fragmentation and enhances the extent of mitophagy. Notably, inhibition/alteration of mitophagy can contribute to myofiber degeneration and weakness in muscle disorders characterized by accumulation of abnormal mitochondria and inclusions ${ }^{30}$.

A different mechanism by which cells may eliminate damaged mitochondria is mitoptosis. In the case of a drop in cellular energy in which mitochondrial stress leads to impaired oxygen utilization, ROS generation increases causing mitochondrial network fragmentation, clustering of damaged mitochondria in the perinuclear region, incorporation into a single-membranedmitoptotic body, and finally extrusion of the mitoptotic body via exocytosis or blebbing ${ }^{31}$. These data are in agreement with the recent evidence that $\mathrm{C} 2 \mathrm{C} 12$ cells can release in the extracellular space microvesicles carrying mt $\mathrm{DNA}^{32}$. To date, it is not clear whether this path is independent or synergic with autophagy. Lyamzaev et al. discovered that mature mitoptotic bodies were not associated with autophagosomes, suggesting that in the setting of whole-cell energy catastrophe, mitoptosis could be more of a parsimonious mechanism for mitochondrial clearing than mitophagy ${ }^{31}$. Once damaged organelles are eliminated, a new mitochondrial pool needs to be generated by mitochondrial biogenesis.

Mitochondriogenesis may be initiated at the same time as autophagy or secondary to subsequent autophagic signaling. A number of signals such as ROS, calcium, energy status, and others influence the activation of the nuclear coactivator PGC-1 $\alpha$ and the associated nuclear respiratory factors ${ }^{33}$. The PGC- $1 \alpha$ transcriptional coactivator is a major regulator of energy metabolism. The latter effects of PGC$1 \alpha$ are likely to represent a compensatory response where it plays a central role in the adaptation of cellular energy metabolism and mitochondrial biogenesis. Hence, mitochondrial homeostasis is reestablished, and the cell is able to avoid bioenergetic failure and cell death. With respect to this issue, building on our group's previous research ${ }^{15}$, we have recently addressed the problem of the role of PGC-1 $\alpha$ in $\mathrm{C} 2 \mathrm{C} 12$ myoblasts subjected to oxidative stress during the early stages of differentiation. In particular, we examined the effect of a mildly toxic concentrations of exogenously added $\mathrm{H}_{2} \mathrm{O}_{2}(0.3 \mathrm{mM})$ on the regulation of PGC-1 $\alpha$ expression and its relationship with AMP-activated protein kinase (AMPK) activation (unpublished observations). In agreement with $\mathrm{Kang}^{34}$ and Irrcher ${ }^{35}$, we found that $1 \mathrm{~h}$ treatment with $\mathrm{H}_{2} \mathrm{O}_{2}$ markedly increased PGC- $1 \alpha$ mRNA expression. It is noteworthy that, concurrently, we also found an increased phosphorylation of AMPK compared to untreated cells, suggesting that oxidative stress induces PGC-1 $\alpha$ through the AMPK signaling pathway. However, despite the fact that challenged C2C12 my- 
oblasts rapidly activate a defense-oriented signaling cascade, they displayed a $30-40 \%$ reduction in their viability as well as a survivors' reduced differentiative efficiency during the post-challenge incubation stage (up to 7 days of culture). This observation implies that, in addition to probably being an obligatory and physiological response to ROS, activation of AMPK and of PGC-1 $\alpha$ may not be sufficient to afford a complete protection to cells against overwhelming oxidative stress. Thus increased mitochondrial production of ROS is involved at multiple levels in promoting apoptosis in skeletal muscle cells, an event which is part of the etiology and progression of numerous pathologies including sarcopenia and muscle disuse atrophy, as well as aging ${ }^{36}$. Romanello et al. ${ }^{37}$ provided direct evidence of the importance of the existence of mitochondrial fission as an amplifying loop in atrophying muscles. The mitochondrial network fragmentation induces energy unbalance, which activates a FoxO3-dependent atrophy program through the AMPK pathway. Thus, mitochondria play a crucial role in catabolic muscle signaling: the mitochondrial fragmentation activates the AMPK-FoxO3 axis, which induces expression of atrophy-related genes, protein breakdown and muscle loss.

Mitochondria are about cellular life and death, as Apostolova N, Blas-Garcia A, Esplugues JV suggest$\mathrm{ed}^{38}$, they are directly involved in triggering different and complexly interconnected programs promoting cell survival or death. In muscle cells mitochondria are involved in apoptosis because they contain several proapoptotic proteins that can lead to cell death upon release into the cytosol. Chronic muscle disuse induced by denervation, bed rest, sedentary life style, aging and mitochondrial impairment leads to an increased susceptibility to mitochondrial mediated apoptosis, whereas exercise may reduce mitochondrially mediated cell death ${ }^{39}$. The mitochondrial electron transport chain is the primary source of reactive oxygen species within the cell. ROS are involved in apoptosis because they change the conformation of mitochondrial permeability transition pore (mtPTP) components to facilitate the release of proapoptotic proteins and induce cytochrome c (c) release from the inner membrane and/or the mitochondrial apoptosis-inducing channel (MAC). Proapoptotic proteins released from SS or IMF mitochondria can initiate either a caspase-dependent or caspase-independent cell death pathway.

\section{Mitochondrial isolation method}

Most of the molecular and biochemical studies of muscle mitochondria have been performed on isolated organelles. Numerous isolation and fractionation procedures applied to mitochondria as well as their membrane-bound sub-compartments together with the identification of specific markers have been reported. One of the major problems in the study of human muscle mitochondria is the difficulty in obtaining sufficient amount of fresh tissue, particularly from healthy human controls. Isolated SSM and IFM mitochondria have characteristic lamelliform and tubular cristae, respectively. The interdependence of mitochondrial morphology and function, demonstrated using isolation methods (the gold standard for the study of mitochondrial function) force us to reconsider the functional characteristics of isolated organelles. Isolated mitochondrial preparations from skeletal muscle cells might present several limiting factors in experimental preparations. Recent evidence shows that the fragmented mitochondrial shapes resulting from routine mitochondrial isolation procedures alters key indices of function in a similar way to that which occurs when mitochondria undergo fission in vivo ${ }^{40}$. Mitochondria can be efficiently isolated by centrifugation and several other techniques, such as free flow electrophoresis ${ }^{41}$. Our experience in isolating mitochondria is specific to cultured muscle cells ${ }^{14}$. About $3.0 \times 10^{7} \mathrm{C} 2 \mathrm{C} 12$ of myoblasts or myotubes have to be harvested and washed with $1 \times$ PBS buffer. In the case of tissues, about $1 \mathrm{gr}$ of tissue is necessary; both samples need to be homogenized. The pellet can be re-suspended in $5 \mathrm{~mL}$ of an ice-cold solution containing $5 \mathrm{mM} \mathrm{K-Hepes,} \mathrm{pH} 7.4$, $210 \mathrm{mM}$ mannitol, $1 \mathrm{mM}$ EGTA, $70 \mathrm{mM}$ sucrose, and $55 \mathrm{ug} / \mathrm{mL}$ digitonin and homogenized by 10 strokes in an ice-cold glass homogenizer. Non-lysed cells and nuclei were pelleted by centrifugation at $600-800 \mathrm{~g}$ for $20 \mathrm{~min}$ at $4^{\circ} \mathrm{C}$, and the supernatant was centrifuged again at $10,000-25,000 \mathrm{~g}$ for $15 \mathrm{~min}$ at $4^{\circ} \mathrm{C}$. The resulting mitochondrial pellet was re-suspended in lysis buffer for further biochemical analyses. However, the mitochondrial fractions contain several contaminations from subcellular organelles, such as endoplasmic reticulum and lysosomes. To diminish contaminants, mitochondria may be further purified using density gradient centrifugation with sucrose, nycodenz, percoll or metrizamide ${ }^{42}$.

\section{Proteomic mapping of mitochondria}

Proteomics is an attractive tool for the study of mitochondria since it allows us to compare the protein expression profile and protein modifications in tissues, cells or organelles under different conditions. The basic procedure for comparative mitochondrial proteomics research includes mitochondria isolation, large-scale separation of mitochondrial proteins and protein identification by mass spectrometry.

Mitochondrial proteomics research includes gel-based and gel-free strategies. The standard gel-based strategy consists in two dimensional gel electrophoresis (2-DE), in which proteins are separated in the first dimension (isoelectrofocusing, IEF), according to their isoelectic point $(\mathrm{pl})$, on immobilized $\mathrm{pH}$ gradient strips and then, in the second dimension, according to molecular weight $(\mathrm{Mw})$ on conventional SDS-PAGE. For isoelectric focusing, the choice of an optimal protocol for sample preparation is critical. The native sample 
has to be converted into a suitable physicochemical state for IEF preserving the native charge and Mw of the proteins. In most cases, the proteins of the sample under study need to be solubilized, disaggregated, denaturated and reduced.

In our studies mitochondria were re-suspended in urea lyses buffer composed of: $8 \mathrm{M}$ urea, $4 \%$ CHAPS, $65 \mathrm{mM}$ DTE and $40 \mathrm{mM}$ Tris base and sonicated for 5 $s$ on ice; after centrifugation the proteins in the supernatant were separated by 2-DE. Proteins separated by electrophoresis and intended for in gel digestion can be visualized by using coomassie brilliant blue, silver nitrate or fluorescent dyes depending on the amount of protein in the sample. Gel images are analyzed with bioinformatic tools to find the differentially expressed protein spots, which are subsequently excised from the gel and subjected to in-gel digestion with trypsin. Finally, the obtained peptides are analyzed by mass spectrometry to identify the excised protein. The approach described above, though it has some limitations, also has clear advantages such as robustness and technical confidence ${ }^{43}$.

\section{Mitochondrial proteomics applied to muscle cell differentiation}

Mitochondria act as a potential regulator of myogenesis. Several studies have demonstrated that mitochondria are involved in regulating myogenic differentiation possibly by myogenin, c-Myc, and calcineurin target $^{14,16,44}$. In fact, when myoblasts are induced to differentiate in the presence of mitochondrial inhibitors, myogenic differentiation is blocked.

In our investigations, we found that myogenesis appears to be dependent on mitochondrial function and mitochondrial biogenesis, as indicated by the rapid increase in mitochondrial mass/volume, mtDNA copy number, mitochondrial enzyme activities, and mRNA levels within the first $48 \mathrm{hrs}$ of myoblast differentiation ${ }^{14}$. These observations are also in agreement with $\mathrm{Kraft}^{45}$. In this analysis we integrated the mitochondrial changes observed by multiple key determinants using proteomic analysis, presenting the first proteomic profile of mitochondria during the myogenesis program. We used a two dimensional electrophoresis, analyzing the differentially expressed proteins in the mitochondrial proteome profile during the myogenic process. We found that enzymes involved in oxidative phosphorylation, such as pyruvate dehydrogenase, malate dehydrogenase, fumaratehydratase, aconitase, and more markedly HB and 5B ATP synthase subunits, increased linearly with mitochondrial biogenesis showing a positive correlation $\left(r^{2}=0.915\right)$. These findings are consistent with the increase in mitochondrial function and membrane depolarization. Moreover, they are in agreement with the increase of mitochondrial cristae observed and the reliance on aerobic metabolism rather than glycolytic metabolism, which characterizes undifferentiated myoblasts. Among the mito- chondrial proteins differentially expressed, Manganese Superoxide Dismutase (MnSOD) is one of the notable proteins involved in the crosstalk between nuclei and mitochondria. It has recently been described as a cell protection molecule whose role is the maintenance of myoblast mitochondrial function and the preservation of potential myoblast stem cell differentiation 46 .

In our model, the increment of MnSOD expression is highlighted during myotube formation (DifD4 - Dif D7). Voltage dependent anion-selective channel protein 1 is another protein associated with myoblast differentiation. It has been recognized as a key protein in mitochondria-mediated apoptosis, since it is a target for the pro- and antiapoptotic Bcl2-family of proteins, and for its function in releasing apoptotic proteins located in the intermembrane space ${ }^{47}$. Apoptosis, which eliminates cells with defects or undergoing damage during differentiation, is required for normal skeletal muscle development ${ }^{48,49}$. The detection of mitochondrial myogenesis-correlated proteins, known to play a role in apoptosis, supports the link between differentiation and this type of cell death ${ }^{50}$. Our investigations did not yield any key proteins up or down regulated during the myogenic process and required for the regulation of basal levels of mitophagy. This is an important process that has received growing attention, especially in the context of muscle development in skeletal muscle ${ }^{37}$, but very little is known about the key proteins involved and the signals required for the regulation of basal levels of mitophagy.

In a previous work we demonstrated that a mildly toxic treatment with $\mathrm{H}_{2} \mathrm{O}_{2}$ impaired the ability of myoblasts to differentiate into mature myotubes ${ }^{15}$, an event that is considered as pathologically relevant in specific human pathologies. In order to evaluate the effects of oxidative stress specifically on mitochondrial protein expression we compared the 2D maps of mitochondria purified from differentiating $\mathrm{C} 2 \mathrm{C} 12$ exposed or not exposed to $\mathrm{H}_{2} \mathrm{O}_{2}$. The oxidant was given during the early stage of the myogenic process; cells were exposed for $1 \mathrm{~h}$ to $0.3 \mathrm{mM} \mathrm{H}_{2} \mathrm{O}_{2} 24 \mathrm{hr}$ after the onset of differentiation and then cultured for 2 more days in fresh, $\mathrm{H}_{2} \mathrm{O}_{2}$-free differentiation medium. Mitochondria were collected before treatment (day 1 of differentiation) and after 2 days (third day of differentiation), together with the control.

The comparison of 2D maps revealed that mitochondria purified from oxidatively injured cells (Fig. 5b), 2 days after treatment, had a profile of protein expression similar to that observed in mitochondria purified from myoblasts (Fig. 5a), except for the MnSOD whose expression was increased by 3.7 times (Spot 12 in Fig. 5c). This suggests that oxidative stress blocked the differentiation process and stimulated the over expression of the detoxifying enzyme. A study of Lee et al. ${ }^{46}$ focused on the effects of MnSOD on myoblast function in cells derived from both young and old mice, which under- or over-express the enzyme. They observed that deficient MnSOD myoblasts impaired proliferation and differentiation capacities; 

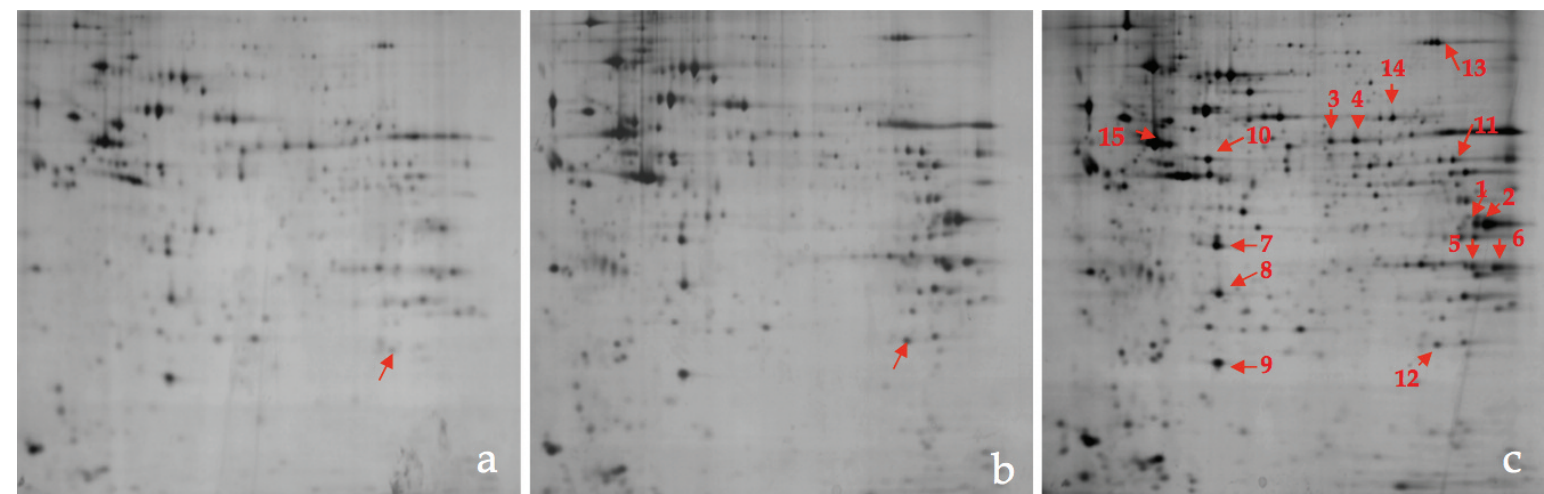

Figure 5. Proteomic mapping from mitochondria purified from confluent $\mathrm{C} 2 \mathrm{C} 12$ cultures subjected to 2-DE before (a) or 3 days after switching to differentiation medium (b-c); b: cells exposed for $1 \mathrm{~h}$ to $0.3 \mathrm{mM} \mathrm{H}_{2} \mathrm{O}_{2} 24 \mathrm{hr}$ after the beginning of differentiation and then cultured for another 2 days in fresh, $\mathrm{H}_{2} \mathrm{O}_{2}$-free differentiation medium; c: control cells at 3 days of differentiation time. Spot numbering corresponds to proteins differentially expressed during the $\mathrm{C} 2 \mathrm{C} 12$ myogenic process and identified as described in Barbieri et al. ${ }^{14}$.

while overexpression of MnSOD preserved myoblast differentiation potential and mitochondrial mass, despite high levels of intracellular $\mathrm{H}_{2} \mathrm{O}_{2}$.

\section{Mitochondrial proteomics applied to exercise re- search}

Several researchers have focused their attention on the characterization of mitochondrial proteins expressed in diseased and healthy tissues. These studies have yielded interesting information leading to important advances in our understanding of mitochondrial functions and cognate molecular signaling. It is recognized that exercise causes physiological adaptations that depend on the duration, frequency, intensity of the physical activity, and these adaptations ultimately result in increased oxygen consumption ${ }^{51,52}$. Since mitochondria are the most important element in oxidative metabolism, it is not surprising that a large range of phenotype modulations associated with exercise occurs on mitochondria.

The first observation, that adaptation to regular endurance exercise increases skeletal muscle oxidative capacity and enhances mitochondrial function, was reported several years ago by Holloszy ${ }^{53}$. In recent years, mitochondria have become the subject of studies aiming to understand the molecular mechanisms of skeletal muscle plasticity in healthy and disease states. Proteomic approaches have identified alterations in mitochondrial protein abundance; indicating defects in mitochondrial metabolism in the skeletal muscle of obese, type 2 diabetic (T2D), and elderly individuals ${ }^{54}$. To date, relatively little is known about the global changes in the mitochondrial proteome following exercise training. Egan et al. ${ }^{55}$ investigated the remodeling of the mitochondrial proteome from human skeletal muscle in response to 14 consecutive days of endurance exercise training, using 2-D DIGE. They analyzed mitochondria-enriched protein fractions from human vastus lateralis muscles taken by biopsy from healthy males. The volunteers, physically inactive for at least six months, were subjected to a protocol consisting in cycling for 60 min per session at $80 \% \mathrm{VO}_{2 \max }$ for 14 consecutive days. Three biopsies were taken from each participant: the first before training, the second and the third after the $7^{\text {th }}$ day and $14^{\text {th }}$ day, respectively. The short-term endurance training determined an extensive remodeling of the mitochondrial proteome in human skeletal muscle, suggesting increased capacity for ATP supply, oxygen delivery and antioxidant capacity, and alterations in the molecular regulatory machinery. Several studies have documented a decline in mitochondrial function with aging, but the physiological relevance of this decline is not clear. Ghosh et al. ${ }^{56}$ examined the mitochondrial proteome (mitochondria were isolated from human vastus lateralis) in young and old healthy sedentary subjects to determine whether the decreases in mitochondrial function observed in old mitochondria were associated with changes in the content of mitochondrial proteins. They found that the content of several electron transport proteins was reduced in the older subjects; in addition, the expression of PGC-1 $\alpha$, NRF-1, and T-FAM also was also reduced in older subjects. Remarkably, the investigation also found that a 16-week aerobic exercise program reversed the mitochondrial phenotype (proteome and function) of old mitochondria. This finding suggests that exercise training induces a mitochondrial remodeling, which provides a mechanistic basis for physical activity in counteracting age related decline in muscle function and inactivity-associated metabolic disease progression.

It is well established that contracting skeletal muscle produces free radicals and it is now clear that both reactive oxygen species and reactive nitrogen 
species (RNS) play important roles in cell signaling pathways involved in muscle adaptation to exercise. However, it is important to observe that excessive ROS production can negatively influence exercise performance and can also be detrimental to health in the long term ${ }^{57}$. Mitochondria are commonly cited as the primary source of superoxide production in contracting muscles; however, recent research suggests that this may not be the case ${ }^{58}$. In addition to mitochondria, in muscle there are other potential cellular sites for ROS production such as nicotinamide adenine dinucleotide phosphate (NADPH) oxidases (sarcoplasmic reticulum, sarcolemma and transverse tubules) and xantine oxidase, as briefly reported above. The site of ROS production in muscle may differ between contracting fibers and fibers exposed to prolonged inactivity; in fact, Kavazis ${ }^{59}$ reported that mitochondrial ROS production largely increases following prolonged periods of inactivity. Further investigation is needed to understand the actual role of mitochondria in ROS and RNS production during muscle contraction.

Exercise is therefore recognized as an important agent in improving health, and it is also an auxiliary in numerous medical treatments and therapy. These findings regarding exercise have led to a better understanding of the plasticity of skeletal muscle, mainly through the modulation of the mitochondrial proteome providing insights into muscle sensitivity to exercise stimulus. All of these results clearly show that phenotype changes associated with exercise are linked directly to mitochondrial proteome modulation. This fact makes mitochondrial research a promising field in sports and medical science.

\section{Mitohormesis links physical exercise and reactive oxygen species generation}

Physical exercise consists in planned, structured, and repetitive bodily movement produced by the contraction of skeletal muscles resulting in a substantial increase in caloric requirement over resting energy expenditure ${ }^{60,61}$.

Physical exercise and chronic contractile activity (CCA) induce a number of physiological adaptations that ameliorate muscle function and exercise performance ${ }^{62}$. Trained muscle undergoes a remodeling toward a more oxidative phenotype altering the ultrastructural and subcellular organization. These modifications include an increase in muscle capillarity, extended intramyocellular glycogen and lipid storage and enhanced insulin responsiveness. However, one of the most important effects of endurance training is mitochondrial biogenesis with an increase in mitochondrial content after only a few weeks of training63,64. Biogenesis may be mediated by metabolic target activation such as AMPK $^{65}$. The increased mitochondrial content and mitochondrial efficiency ameliorate the cellular antioxidant defense capacity and insulin sensitivity ${ }^{66}$. It is well- known that physical exercise increases ROS generation in skeletal muscle cells ${ }^{7}$. However, the sources of the generation of ROS have not yet been explained Jackson et al., 2007. During exercise, the increased ROS production is mainly due to the high oxygen consumption that takes place during increased mitochondrial activity, although recent studies have demonstrated ${ }^{7}$ several non mitochondrial sources for the generation of ROS within skeletal muscle such as the plasma membrane and endomembranes $\operatorname{NADP}(\mathrm{H})$ oxidases $^{8}$. These studies suggest that there is probably generation of ROS at multiple subcellular locations in response to a multiplicity of mechanical and metabolic stimuli in muscle cells. Recently, it has been demonstrated that exercise-induced ROS function as signaling molecules that contribute to some of the adaptations occurring after physical exercise, including an increase in endogenous antioxidant defense capacity and insulin sensitivity. This adaptive response to ROS, often called mitochondrial hormesis or mitohormesis, has emerged as a novel and key component not only for physical exercise adaptation but also for caloric restriction and insulin/Insulin like Growth Factor 1 signaling ${ }^{67}$. Mitohormesis links physical exercise and the consequent formation of reactive oxygen species to insulin sensitivity and antioxidant defense as well as caloric restriction. Generating ROS by chronic physical activity or caloric restriction: i) stimulates mitochondrial signaling to the cell, ii) reduces glucose metabolism, iii) induces mitochondrial metabolism, iv) increases stress resistance and generally prolongs lifespan ${ }^{66}$.

Physical exercise induces ameliorating effects on insulin resistance by increasing mitochondrial reactive oxygen species generation in skeletal muscle to stimulate expression of PGCs as insulin sensitivity inducers, as well as SOD 1, 2 and glutathione peroxidase, strategic enzymes of ROS defense. Remarkably, by blocking exercise-dependent ROS with antioxidant supplements, the health benefits of physical exercise are lost; physical exercise fails to promote insulin sensitivity and antioxidant defense in the presence of vitamin $\mathrm{C}$ and vitamin $\mathrm{E}^{66}$.

The evidence is ambiguous in this regard with a number of findings suggesting that exposure to ROS may promote insulin resistance, whereas others suggest that such exposure may have the opposite effect ${ }^{68,69}$. Previous works also contain contradictory findings ${ }^{70}$. One possible reason for the divergences among the various studies may stem from the inverse relation between ROS and insulin sensitivity obtained in models of continuous exposure to increased ROS levels ${ }^{69}$, whereas current findings ${ }^{64}$ may imitate transient increases in ROS generation during limited periods of physical exercise.

This evidence suggests that activation of mitochondrial metabolism may promote metabolic health and potentially prolong lifespan. Like calorie and/or glucose (and possibly amino acid) restriction, physical exercise also induces mitochondrial metabolism activation 
and transient ROS formation 7,58 . Interestingly, it has been demonstrated that supplementation with antioxidants prevents ${ }^{66}$ the health benefits of physical exercise $^{71}$. This suggests that physical exercise shares common metabolic paths with caloric restriction and glucose restriction increasing mitohormesis and inducing a positive adaptive response that ends with stress resistance, antioxidant defense and prolonged life span.

\section{Conclusions}

This review has examined the major cytochemical, molecular biological, and biochemical techniques used in investigating muscle mitochondria adaptation in oxidative stress conditions. Molecular and proteomic techniques, which are steadily improving, may provide us a better understanding of these processes. Moreover, the use of such technologies may provide insights into physiology yielding practical benefits such as the improvement of biotechnological research related to life style design and physical activity prescription in a wide array of physiological and pathophysiological conditions in which mitohormesis may play a central role.

\section{Acknowledgments}

The authors wish to thank the Istituto Interuniversitario di Miologia (IIM) members for suggestions; Prof. Elisabetta Falcieri, Dr. Michela Battistelli and Dr. Sara Salucci, Department of Earth, Life and Environmental Sciences of the University of Urbino, Italy, for their kind support; Prof. Timothy Bloom, Centro Linguistico d'Ateneo of the University of Urbino, Italy, for a critical reading of the manuscript.

\section{References}

1. Boncompagni S, Rossi AE, Micaroni M, et al. Mitochondria are linked to calcium stores in striated muscle by developmentally regulated tethering structures. Mol Biol Cell 2009; 20(3):1058-1067.

2. De Stefani D, Raffaello A, Teardo E, Szabo I, Rizzuto R. A forty-kilodalton protein of the inner membrane is the mitochondrial calcium uniporter. Nature $2011 ; 476(7360): 336-$ 340.

3. Nunnari J, Suomalainen A. Mitochondria: in sickness and in health. Cell 2012 16; 148(6):1145-1159.

4. Schapira AH. Mitochondrial diseases. Lancet 2012; 379(9828):1825-1834.

5. Sestili P, Paolillo M, Lenzi M, et al. Sulforaphane induces DNA single strand breaks in cultured human cells. Mutat Res 2010; 689(1-2):65-73.

6. Muller FL, Liu Y, Van Remmen H. Complex III releases superoxide to both sides of the inner mitochondrial membrane. J Biol Chem 2004; 279(47):49064-49073.

7. Powers SK, Jackson MJ. Exercise-induced oxidative stress: cellular mechanisms and impact on muscle force production. Physiol 2008; 88(4):1243-1276.

8. Barbieri E, Sestili P. Reactive oxygen species in skeletal muscle signaling. J Signal Transduct 2012; 2012:982794.

9. Smith MA, Reid MB. Redox modulation of contractile function in respiratory and limb skeletal muscle. Respir Physiol Neurobiol 2006; 151(2-3):229-241.

10. Marin-Garcia J. Mitochondria and their role in cardiovascular disease. New York: Springer; 2012.

11. Gomes A, Fernandes E, Lima JL. Fluorescence probes used for detection of reactive oxygen species. J Biochem Biophys Methods 2005; 65(2-3):45-80.

12. Owusu-Ansah E, Yavari A, Banerjee U. A protocol for_in vivo_detection of reactive oxygen species. 2008.

13. Luchetti F, Canonico B, Mannello F, et al. Melatonin reduces early changes in intramitochondrial cardiolipin during apoptosis in U937 cell line. Toxicol In Vitro 2007; 21(2):293301.

14. Barbieri E, Battistelli M, Casadei L, et al. Morphofunctional and Biochemical Approaches for Studying Mitochondrial Changes during Myoblasts Differentiation. J Aging Res 2011; 2011:845379.

15. Sestili $P$, Barbieri E, Martinelli C, et al. Creatine supplementation prevents the inhibition of myogenic differentiation in oxidatively injured $\mathrm{C} 2 \mathrm{C} 12$ murine myoblasts. Mol Nutr Food Res 2009; 53(9):1187-1204.

16. Rochard P, Rodier A, Casas F, et al. Mitochondrial activity is involved in the regulation of myoblast differentiation through myogenin expression and activity of myogenic factors. J Biol Chem 2000; 275(4):2733-2744.

17. Pizzo P, Drago I, Filadi R, Pozzan T. Mitochondrial $\mathrm{Ca}(2)(+)$ homeostasis: mechanism, role, and tissue specificities. Pflugers Arch 2012; 464(1):3-17.

18. Franzini-Armstrong $C$. ER-mitochondria communication. How privileged? Physiology (Bethesda) 2007; 22:261-268.

19. de Brito OM, Scorrano L. Mitofusin 2 tethers endoplasmic reticulum to mitochondria. Nature 2008; 456(7222): 605 610.

20. Connor MK, Bezborodova O, Escobar CP, Hood DA. Effect of contractile activity on protein turnover in skeletal muscle mitochondrial subfractions. J Appl Physiol (1985). 2000; 88(5):1601-1606.

21. Frank M, Duvezin-Caubet S, Koob S, et al. Mitophagy is triggered by mild oxidative stress in a mitochondrial fission dependent manner. Biochim Biophys Acta 2012; 1823(12):2297-2310.

22. Schiaffino S, Dyar KA, Ciciliot S, Blaauw B, Sandri M. Mechanisms regulating skeletal muscle growth and atrophy. FEBS J. 2013; 280(17):4294-4314.

23. Hales KG. The machinery of mitochondrial fusion, division, and distribution, and emerging connections to apoptosis. Mitochondrion 2004; 4(4):285-308.

24. Dagda RK, Cherra SJ, 3rd, Kulich SM, Tandon A, Park D, Chu CT. Loss of PINK1 function promotes mitophagy through effects on oxidative stress and mitochondrial fission. J Biol Chem 2009; 284(20):13843-13855.

25. Gomes LC, Scorrano L. Mitochondrial morphology in mitophagy and macroautophagy. Biochim Biophys Acta 2013; 1833(1):205-212.

26. Narendra D, Tanaka A, Suen DF, Youle RJ. Parkin is recruited selectively to impaired mitochondria and promotes their autophagy. J Cell Biol 2008; 183(5):795-803.

27. Zhang J, Ney PA. Role of BNIP3 and NIX in cell death, autophagy, and mitophagy. Cell Death Differ 2009; 16(7):939946.

28. Okamoto K, Kondo-Okamoto N. Mitochondria and autophagy: critical interplay between the two homeostats. Biochim Biophys Acta 2012; 1820(5):595-600.

29. Mammucari C, Rizzuto R. Signaling pathways in mitochondrial dysfunction and aging. Mech Ageing Dev. 2010; 131(7-8):536-543. 
30. Masiero E, Agatea L, Mammucari C, et al. Autophagy is required to maintain muscle mass. Cell Metab 2009; 10(6):507-515.

31. Lyamzaev KG, Nepryakhina OK, Saprunova VB, et al. Novel mechanism of elimination of malfunctioning mitochondria (mitoptosis): formation of mitoptotic bodies and extrusion of mitochondrial material from the cell. Biochim Biophys Acta 2008; 1777(7-8):817-825.

32. Guescini M, Guidolin D, Vallorani L, et al. C2C12 myoblasts release micro-vesicles containing mtDNA and proteins involved in signal transduction. Exp Cell Res 2010; 316(12):1977-1984.

33. Kelly DP, Scarpulla RC. Transcriptional regulatory circuits controlling mitochondrial biogenesis and function. Genes Dev 2004; 18(4):357-368.

34. Kang C, O'Moore KM, Dickman JR, Ji LL. Exercise activation of muscle peroxisome proliferator-activated receptor-gamma coactivator-1alpha signaling is redox sensitive. Free Radic Biol Med 2009; 47(10):1394-1400.

35. Irrcher I, Ljubicic V, Hood DA. Interactions between ROS and AMP kinase activity in the regulation of PGC-1alpha transcription in skeletal muscle cells. Am J Physiol Cell Physiol 2009; 296(1):C116-123.

36. Musaro A, Fulle S, Fano G. Oxidative stress and muscle homeostasis. Curr Opin Clin Nutr Metab Care 2010; 13(3):236-242.

37. Romanello V, Guadagnin E, Gomes L, et al. Mitochondrial fission and remodelling contributes to muscle atrophy. EMBO J 2010; 29(10):1774-1785.

38. Apostolova N, Blas-Garcia A, Esplugues JV. Mitochondria sentencing about cellular life and death: a matter of oxidative stress. Curr Pharm Des 2011; 17(36):4047-4060.

39. Adhihetty PJ, O'Leary MF, Hood DA. Mitochondria in skeletal muscle: adaptable rheostats of apoptotic susceptibility. Exerc Sport Sci Rev 2008; 36(3):116-121.

40. Picard M, Taivassalo T, Gouspillou G, Hepple RT. Mitochondria: isolation, structure and function. J Physiol 2011; 589(Pt 18):4413-4421.

41. Zischka H, Weber G, Weber PJ, et al. Improved proteome analysis of Saccharomyces cerevisiae mitochondria by freeflow electrophoresis. Proteomics 2003; 3(6):906-916.

42. Chen X, Wei S, Yang F. Mitochondria in the pathogenesis of diabetes: a proteomic view. Protein Cell 2012; 3(9):648660.

43. Rabilloud T, Chevallet M, Luche S, Lelong C. Two-dimensional gel electrophoresis in proteomics: Past, present and future. J Proteomics 2010; 73(11):2064-2077.

44. Seyer P, Grandemange S, Busson M, et al. Mitochondrial activity regulates myoblast differentiation by control of c-Myc expression. J Cell Physiol 2006; 207(1):75-86.

45. Kraft CS, LeMoine CM, Lyons CN, Michaud D, Mueller CR, Moyes $\mathrm{CD}$. Control of mitochondrial biogenesis during myogenesis. Am J Physiol Cell Physiol 2006; 290(4):C11191127.

46. Lee S, Van Remmen H, Csete M. Sod2 overexpression preserves myoblast mitochondrial mass and function, but not muscle mass with aging. Aging Cell 2009; 8(3):296-310.

47. Shoshan-Barmatz V, Israelson A, Brdiczka D, Sheu SS. The voltage-dependent anion channel (VDAC): function in intracellular signalling, cell life and cell death. Curr Pharm Des 2006; 12(18):2249-2270.

48. Burattini S, Battistelli M, Falcieri E. Morpho-functional features of in-vitro cell death induced by physical agents. Curr Pharm Des 2010; 16(12):1376-1386.

49. Sandri M, Carraro U. Apoptosis of skeletal muscles during development and disease. Int J Biochem Cell Biol 1999; 31(12):1373-1390.

50. Casadei L, Vallorani L, Gioacchini AM, et al. Proteomics- based investigation in $\mathrm{C} 2 \mathrm{C} 12$ myoblast differentiation. Eur J Histochem 2009; 53(4):261-268.

51. Guescini M, Fatone C, Stocchi L, et al. Fine needle aspiration coupled with real-time PCR: a painless methodology to study adaptive functional changes in skeletal muscle. Nutr Metab Cardiovasc Dis 2007; 17(5):383-393.

52. Hawley JA. Molecular responses to strength and endurance training: are they incompatible? Appl Physiol Nutr Metab 2009; 34(3):355-361.

53. Holloszy JO. Biochemical adaptations in muscle. Effects of exercise on mitochondrial oxygen uptake and respiratory enzyme activity in skeletal muscle. J Biol Chem 1967; 242(9):2278-2282.

54. Hwang $\mathrm{H}$, Bowen $\mathrm{BP}$, Lefort $\mathrm{N}$, et al. Proteomics analysis of human skeletal muscle reveals novel abnormalities in obesity and type 2 diabetes. Diabetes 2010; 59(1):33-42.

55. Egan B, Dowling $\mathrm{P}, \mathrm{O}$ 'Connor $\mathrm{PL}$, et al. 2-D DIGE analysis of the mitochondrial proteome from human skeletal muscle reveals time course-dependent remodelling in response to 14 consecutive days of endurance exercise training. Proteomics 2011; 11(8):1413-1428.

56. Ghosh S, Lertwattanarak R, Lefort N, et al. Reduction in reactive oxygen species production by mitochondria from elderly subjects with normal and impaired glucose tolerance. Diabetes 2011; 60(8):2051-2060.

57. Sahlin K, Shabalina IG, Mattsson CM, et al. Ultraendurance exercise increases the production of reactive oxygen species in isolated mitochondria from human skeletal muscle. J Appl Physiol (1985). 2010; 108(4):780-787.

58. Powers SK, Talbert EE, Adhihetty PJ. Reactive oxygen and nitrogen species as intracellular signals in skeletal muscle. J Physiol 2011; 589(Pt 9):2129-2138.

59. Kavazis AN, Talbert EE, Smuder AJ, Hudson MB, Nelson WB, Powers SK. Mechanical ventilation induces diaphragmatic mitochondrial dysfunction and increased oxidant production. Free Radic Biol Med 2009; 46(6):842-850.

60. Caspersen CJ, Powell KE, Christenson GM. Physical activity, exercise, and physical fitness: definitions and distinctions for health-related research. Public Health Rep 1985; 100(2):126-131.

61. The President Council on Physical Fitness and Sports. Definitions - Health, Fitness, and Physical Activity. Washington (DC)2000. Available from: http://purl.access.gpo.gov/ GPO/LPS21074.

62. Fitts $\mathrm{RH}$. Effects of regular exercise training on skeletal muscle contractile function. Am J Phys Med Rehabil 2003; 82(4):320-331.

63. Adhihetty PJ, Irrcher I, Joseph AM, Ljubicic V, Hood DA. Plasticity of skeletal muscle mitochondria in response to contractile activity. Exp Physiol 2003; 88(1):99-107.

64. Befroy DE, Petersen KF, Dufour S, Mason GF, Rothman $\mathrm{DL}$, Shulman GI. Increased substrate oxidation and mitochondrial uncoupling in skeletal muscle of endurance-trained individuals. Proc Natl Acad Sci U S A 2008; 105(43):1670116706.

65. Zong $\mathrm{H}$, Ren JM, Young $\mathrm{LH}$, et al. AMP kinase is required for mitochondrial biogenesis in skeletal muscle in response to chronic energy deprivation. Proc Natl Acad Sci U S A 2002; 99(25):15983-15987.

66. Ristow M, Zarse K, Oberbach A, et al. Antioxidants prevent health-promoting effects of physical exercise in humans. Proc Natl Acad Sci U S A 2009; 106(21):8665-8670.

67. Ristow M, Zarse K. How increased oxidative stress promotes longevity and metabolic health: The concept of mitochondrial hormesis (mitohormesis). Exp Gerontol 2010; 45(6):410-418.

68. Goldstein BJ, Mahadev K, Wu X. Redox paradox: insulin action is facilitated by insulin-stimulated reactive oxygen 


\section{E. Barbieri et al.}

species with multiple potential signaling targets. Diabetes 2005; 54(2):311-321.

69. Houstis N, Rosen ED, Lander ES. Reactive oxygen species have a causal role in multiple forms of insulin resistance. Nature 2006; 440(7086):944-948.

70. Schulz TJ, Zarse K, Voigt A, Urban N, Birringer M, Ristow
M. Glucose restriction extends Caenorhabditis elegans life span by inducing mitochondrial respiration and increasing oxidative stress. Cell Metab 2007; 6(4):280-293.

71. Lanza IR, Short DK, Short KR, et al. Endurance exercise as a countermeasure for aging. Diabetes 2008; 57(11):29332942. 